\title{
Design analysis of 2D graphene oxide films integrated with microring resonators for FWM
}

\author{
David J. Moss \\ Optical Sciences Center, Swinburne University of Technology, Hawthorn, VIC 3122, Australia.
}

\begin{abstract}
We theoretically investigate and optimize the performance of four-wave mixing (FWM) in microring resonators (MRRs) integrated with two-dimensional (2D) layered graphene oxide (GO) films. Owing to the interaction between the MRRs and the highly nonlinear GO films as well as to the resonant enhancement effect, the FWM efficiency in GO-coated MRRs can be significantly improved. Based on previous experiments, we perform detailed analysis for the influence of the GO film parameters and MRR coupling strength on the FWM conversion efficiency (CE) of the hybrid MRRs. By optimizing the device parameters to balance the trade-off between the Kerr nonlinearity and loss, we achieve a high $\mathrm{CE}$ enhancement of $\sim 18.6 \mathrm{~dB}$ relative to the uncoated MRR, which is $\sim 8.3 \mathrm{~dB}$ higher than previous experimental results. The influence of photothermal changes in the GO films as well as variations in the MRR parameters such as the ring radius and waveguide dispersion on the FWM performance is also discussed. These results highlight the significantly improved FWM performance that can be achieved in MRRs incorporating GO films and provide a guide for optimizing their FWM performance.
\end{abstract}

Keywords - Four-wave mixing, 2D materials, microring resonator, graphene oxide.

\section{INTRODUCTION}

$\mathrm{G}$ raphene oxide (GO) has become a rising star in the family of two-dimensional (2D) materials owing to its potential for mass production as well as the flexibility in tuning its material properties [1-4]. Recently, the excellent nonlinear optical properties of GO have attracted significant interest [5-9]. It has been reported that GO has an ultrahigh Kerr nonlinearity $\left(n_{2}\right)$ that is about 4 orders of magnitude higher than nonlinear bulk materials such as silicon and chalcogenide glasses $[5,6,10]$. In addition, GO has a large optical bandgap (typically $>2 \mathrm{eV}[1,11]$ ), which yields a material absorption that is over 2 orders of magnitude lower than graphene as well as negligible two-photon absorption (TPA) in the telecom band [12, 13]. Another important advantage of GO is that it can be mass produced from natural graphite [3]. This, together with facile solution-based fabrication processes [14], is attractive for large-scale manufacturing of integrated devices that incorporate GO films $[2,15,16]$.

Based on these advantages, many high performance nonlinear photonic devices that incorporate GO films $[13,17-$
21] have been demonstrated - especially those based on complementary metal-oxide-semiconductor (CMOS) compatible integrated platforms [13, 17-19]. Enhanced fourwave mixing (FWM) in GO-coated doped silica and silicon nitride $(\mathrm{SiN})$ waveguides has been reported [13, 17], where conversion efficiency (CE) enhancements of up to $6.9 \mathrm{~dB}$ and $9.1 \mathrm{~dB}$ relative to the uncoated waveguides were achieved. Significant spectral broadening of optical pulses in GO-coated silicon waveguides induced by self-phase modulation (SPM) has also been observed [19], achieving a high spectral broadening factor of 4.34 for a device with a patterned film including 10 layers of GO. A significant enhancement in the nonlinear figure of merit (FOM) for silicon nanowires by a factor of 20 was also achieved, resulting in a FOM $>5$.

In our previous work [18], we experimentally demonstrated enhanced FWM in CMOS compatible doped silica microring resonators (MRRs) integrated with 2D layered GO films. Due to the resonant enhancement effect $[22,23]$, an increase of up to $\sim 10.3 \mathrm{~dB}$ in the FWM CE was achieved. In this paper, we fully investigate and optimize the FWM performance of GOcoated MRRs based on previous experimental measurements of the GO film properties such as loss and Kerr nonlinearity, which are distinct from bulk materials and show a strong dependence on the film thickness and optical power. We perform a detailed analysis of the influence of the GO film parameters and MRR coupling strength on the FWM CE of the hybrid MRRs. By properly balancing the trade-off between the Kerr nonlinearity and loss, a high CE enhancement of $\sim 18.6 \mathrm{~dB}$ relative to the uncoated MRR is achieved, which is $\sim 8.3 \mathrm{~dB}$ higher than what has been achieved experimentally. We also discuss the influence of photo-thermal changes in the GO films as well as the variation of other MRR parameters such as ring radius and waveguide dispersion on the FWM performance. These results highlight the significant potential to improve on previous experimental results [18] and provide a guide for optimizing FWM performance of MRRs integrated with GO films. 


\section{DEVICE STRUCTURE}

Fig. 1(a) shows a schematic of an integrated MRR made from doped silica, with 1 layer of patterned GO film being coated on the planarized waveguide top surface. Inset shows a schematic illustration for the atomic structure of GO, including different oxygen-containing functional groups (OFGs) such as hydroxyl, epoxide, and carboxylic decorated on a graphene-like carbon lattice. In contrast to graphene, which has a metallic behavior with a zero bandgap [24], pristine $\mathrm{GO}$ is a dielectric material with a bandgap $>2 \mathrm{eV}[1$, $12]$. This is larger than both the single photon $(\sim 0.8 \mathrm{eV})$ and two-photon $(\sim 1.6 \mathrm{eV})$ energies around $1550 \mathrm{~nm}$, resulting in negligible linear light absorption or TPA in the telecom band. We consider MRRs that are fabricated on a high index doped silica glass (Hydex) platform [25] via CMOS compatible processes. More details about the Hydex device fabrication can be found in Refs. [22, 26, 27]. As compared with GOcoated waveguides, GO-coated MRRs can dramatically improve the FWM efficiency by virtue of the resonant enhancement of the optical intensity within the resonant cavities [22, 23], thus significantly reducing the required power. The upper cladding of the doped silica MRR is removed by chemical mechanical polishing (CMP) to obtain a planarized waveguide top surface for GO film coating. The GO film coating can be achieved using a solution-based method that yields layer-by-layer film deposition and precise control of the film thickness with an ultrahigh resolution of $\sim 2$ $\mathrm{nm}[12,28]$. Unlike graphene or other 2D materials that are typically prepared via non-solution-based deposition followed by cumbersome layer transfer processes [29-32], our coating method enables large-area, transfer-free, and high-quality GO film coating with high fabrication stability, mass producibility, and excellent film attachment onto integrated waveguides [2, 19]. Patterning of the films can be achieved via standard
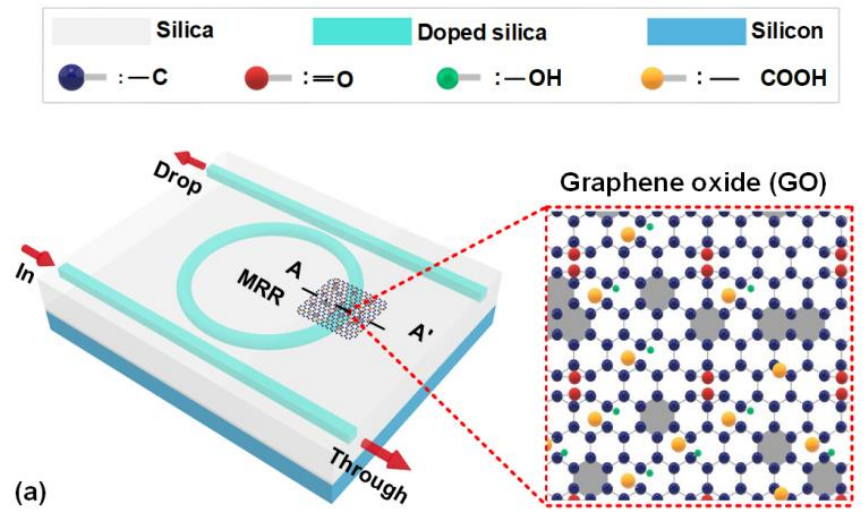

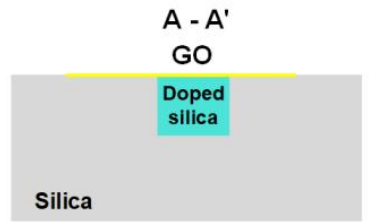

(b)

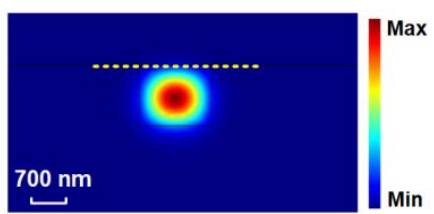

(c)
Fig. 1. (a) Schematic illustration of an integrated doped silica MRR coated with 1 layer of patterned GO film. Inset shows a schematic of atomic structure of GO. (b) Schematic illustration of the cross section of the hybrid MRR in (a). (c) TE mode profile corresponding to (b).

lithography and lift-off processes $[18,28]$. This, together with the layer-by-layer deposition of GO films, forms the basis for the optimization of the FWM performance of the hybrid MRRs with different GO film thicknesses and pattern lengths.

Fig. 1(b) shows a schematic of the waveguide cross section of the hybrid MRR in Fig. 1(a). The corresponding transverse electric (TE) mode profile is shown in Fig. 1(c). We chose the TE polarization in our following analysis because it supports an in-plane interaction between the film and the evanescent field leaking from the MRR, which is much stronger than the

TABLE I

PARAMETERS OF DOPED SILICA MRR, GO FILM, AND CW LASER

\begin{tabular}{|c|c|c|c|c|}
\hline \multirow{4}{*}{$\begin{array}{l}\text { Doped } \\
\text { Silica } \\
\text { MRR }\end{array}$} & \multirow{2}{*}{$\begin{array}{c}\text { Material } \\
\text { parameters }\end{array}$} & Refractive index ${ }^{a}$ & Extinction coefficient & Kerr coefficient $\left(\mathrm{m}^{2} / \mathrm{W}\right)$ \\
\hline & & $n_{\text {hydex }}: 1.7[25]$ & $k_{\text {hydex }}: 0[18]$ & $n_{2-\text { hydex }}: 1.3 \times 10^{-19}[18]$ \\
\hline & \multirow{2}{*}{$\begin{array}{c}\text { Physical } \\
\text { parameters }\end{array}$} & Transmission / coupling coefficients & Radius $^{\mathrm{c}}$ & Propagation loss $(\mathrm{dB} / \mathrm{cm})$ \\
\hline & & $t, \kappa^{\mathrm{b}}$ & $R$ & $0.25[18]$ \\
\hline \multirow{4}{*}{$\begin{array}{l}\text { GO } \\
\text { film }\end{array}$} & \multirow{2}{*}{$\begin{array}{c}\text { Material } \\
\text { parameters }\end{array}$} & Refractive index & Extinction coefficient ${ }^{\mathrm{d}}$ & Kerr coefficient $\left(\mathrm{m}^{2} / \mathrm{W}\right)$ \\
\hline & & $n_{G O}: 1.97[13]$ & $k_{G O}(N): 0.0074-0.0189[18]$ & $n_{2-G O}(N): 1.7 \times 10^{-14}-2.7 \times 10^{-14}[18]$ \\
\hline & \multirow{2}{*}{$\begin{array}{c}\text { Physical } \\
\text { parameters }\end{array}$} & Thickness for 1 layer ${ }^{\mathrm{e}}$ & GO layer number & Coating length \\
\hline & & $d: 2 \mathrm{~nm}[18]$ & $N$ & $L_{c}$ \\
\hline \multirow{2}{*}{$\begin{array}{l}\text { CW } \\
\text { laser }\end{array}$} & \multirow{2}{*}{$\begin{array}{c}\text { Physical } \\
\text { parameters }\end{array}$} & $\mathrm{CW}$ power for loss measurement & Pump power for FWM & Signal power for FWM \\
\hline & & $P_{C W}$ & $P_{p}$ & $P_{s}$ \\
\hline
\end{tabular}

${ }^{a}$ Here we show the refractive indices at $1550 \mathrm{~nm}$, the same applies for other material parameters in this Table.

${ }^{\mathrm{b}} t^{2}+\kappa^{2}=1$ for lossless coupling is assumed for the directional couplers.

c The circumference of the MRR is $L=2 \pi \mathrm{R}$.

${ }^{\mathrm{d}}$ Here we show the extinction coefficient and Kerr coefficient at $P_{C W}=25 \mathrm{dBm}$ for $N=1-50$ based on the measured results in Ref. [18].

${ }^{\text {e }}$ Following our previous experimental measurements [18], the GO film thickness is assumed to be proportional to $N$, with a thickness of $2 \mathrm{~nm}$ per layer. 
out-of-plane interaction due to the significant optical anisotropy of 2D films [28, 31, 32]. Table I summarizes the parameters of the doped silica MRR, the GO film, and the continuous-wave $(\mathrm{CW})$ laser used in our following analysis, with the former two being further classified into material and physical parameters. Four-port MRRs with two identical directional couplers are used in our following analysis, which is consistent with that used in Ref. [18]. The GO-coated MRRs are designed based on, but not limited to, the Hydex platform.

In the following sections, we first investigate the powerdependent propagation loss and nonlinear parameters of the hybrid MRRs induced by photo-thermal changes in the GO films. Next, by properly balancing the trade-off between loss and the Kerr nonlinearity, we optimize the FWM CE in the hybrid MRRs by regulating the GO film parameters $\left(N, L_{c}\right)$ and the MRR coupling strength $(t)$. Finally, we discuss the influence of photo-thermal changes in the GO films as well as the effect of varying other MRR parameters such as the ring radius and waveguide dispersion on the FWM performance of the hybrid MRRs.

\section{POWER-DEPENDENT PROPAGATION LOSS AND NONLINEAR PARAMETERS}

As reported in previous work [18], the linear loss $(k)$ and Kerr nonlinearity $\left(n_{2}\right)$ of GO films coated on integrated waveguides change with input $\mathrm{CW}$ power, particularly at high powers. This can be attributed to photo-thermal changes in the GO films, which is a combined result of power sensitive photo-thermal reduction as well as self-heating and thermal dissipation in the multilayered film structure [17, 18, 33]. Such changes are not permanent and can revert back after the power is turned off. In addition, these changes have a slow time response on the order of millisecond, which is different to FWM and TPA that have ultrafast response times on the order of femtoseconds [17]. Photo-thermal changes in the GO films lead to power-dependent propagation loss and nonlinear parameters for GO-coated waveguides, and this is further amplified in GO-coated MRRs due to resonant enhancement. In this section, we investigate the power-dependent propagation loss and nonlinear parameters of the hybrid MRRs induced by the photo-thermal changes in GO films.

We first calculate the resonant build-up factor $(I)$ of a MRR as a function of its coupling strength $(t)$ and round-trip field transmission factor $(A)$. The $\Gamma$ reflects the relationship between the input $\mathrm{CW}$ power $\left(P_{C W}\right)$ and the intracavity power $\left(P_{\text {intra }}\right)$ in a MRR, which will be used for calculating $P_{\text {intra }}$ directly related to the propagation loss and nonlinear linear parameters of the hybrid MRRs in our following analysis. Fig. 2(a) shows $\Gamma$ versus $t$ and $A$. The $\Gamma$ was calculated at resonant wavelengths based on $[34,35]$ :

$$
\Gamma=\frac{P_{\text {intra }}}{P_{C W}}=\left(1-t^{2}\right) t^{2} A^{2} /\left(1-2 t^{2} A+t^{4} A^{2}\right)
$$

In Eq. (1), $A$ can be further expressed as:

$$
A=\exp \left(-\frac{1}{2} \alpha_{u} L_{u}\right) \exp \left(-\frac{1}{2} \alpha_{c} L_{c}\right)
$$
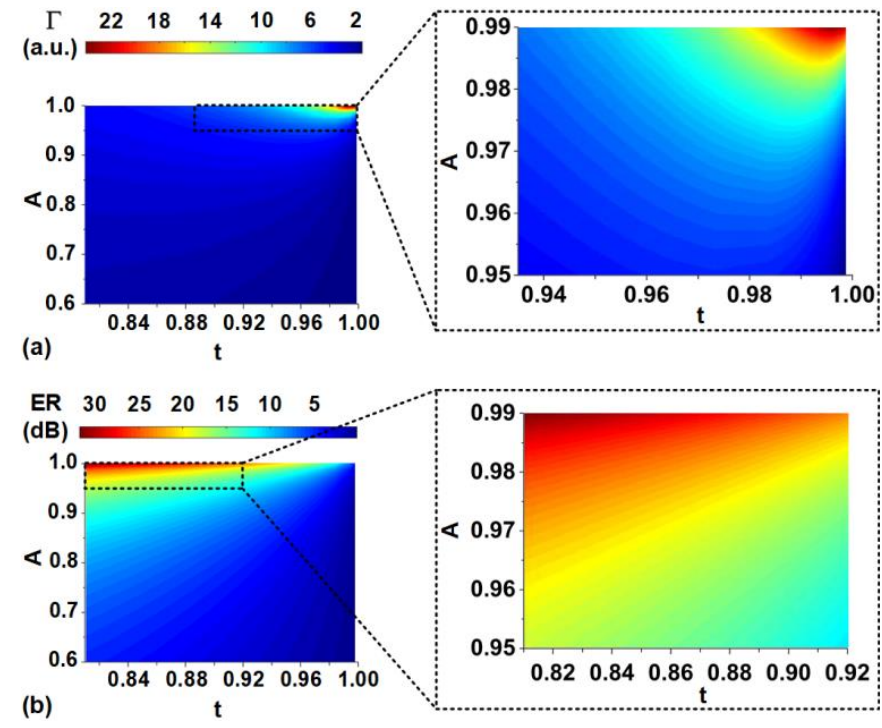

Fig. 2. (a) $\Gamma$ versus $t$ and $A$. (b) ER versus $t$ and $A$. The insets in (a) and (b) show the corresponding zoom-in views.

where $\alpha_{c, u}$ and $L_{c, u}$ are the loss factors and lengths of the GO coated and uncoated waveguide segments, respectively. In Fig. 2(a), the maximum $A$ is 0.989 , which corresponds to the uncoated MRR (unless otherwise specified, the MRR radius used is $592 \mu \mathrm{m}$ - the same as in Ref. [18]). The maximum $\Gamma$ is achieved at $t=0.994$ and $A=0.989$, which is determined by the balance between $t$ and $A$, as reflected by Eq. (1). Fig. 2(b) shows the MRR's extinction ratio (ER) versus $t$ and $A$. The ER increases with $A$ but decreases with $t$, mainly due to the change in the difference between intracavity loss and external coupling loss of a four-port MRR with two identical directional couplers [36-42].

In previous work [18], we measured the propagation loss and nonlinear parameters versus input $\mathrm{CW}$ power $\left(P_{C W}\right)$ for hybrid MRRs with $1-5$ layers of uniformly coated and $10-$ 50 layers of patterned (50- $\mu \mathrm{m}$-long) GO, respectively. The coupling strength of the uncoated MRR was 0.912. In Figs. 3(a) and (b), we fit the measured power-dependent propagation loss and nonlinear parameters as functions of the intracavity power $P_{\text {intra }}$, which will be used for calculating FWM CE in next section. The input CW power $P_{C W}$ in Ref. [18] (from $15 \mathrm{dBm}$ to $25 \mathrm{dBm}$ ) is converted to corresponding intracavity power $P_{\text {intra }}$ based on the calculated $\Gamma$ in Fig. 2(a). In Fig. 3(a), the propagation loss increases with GO layer number $N$. This is mainly due to an increase in mode overlap for the hybrid MRRs with thicker GO films. A small contribution is from an increase in the material absorption arising from inhomogeneous defects and imperfect contact between the multiple GO layers $[13,28]$. As $P_{\text {intra }}$ increases, the hybrid MRRs show an increased propagation loss, in contrast to the uncoated MRR that manifests a constant propagation loss. This further confirms the power sensitive photo-thermal changes in GO films. Following the same trends with the propagation loss, the nonlinear parameter $\gamma$ in Fig. 3(b) increases with both $N$ and $P_{\text {intra. }}$. This reflects the trade-off between the Kerr nonlinearity and linear loss, which 

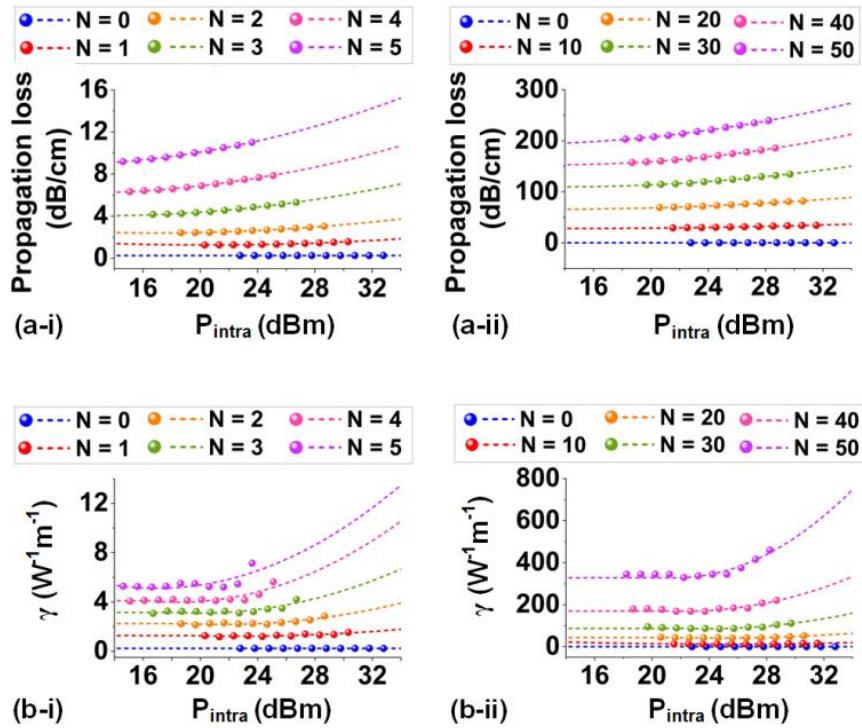

Fig. 3. (a)-(b) Fit propagation loss and nonlinear parameters $\gamma$ of GO-coated MRRs versus intracavity power $P_{\text {intra }}$ based on previous measured results for hybrid MRRs with (i) $1-5$ layers of uniformly coated and (ii) $10-50$ layers of patterned GO, respectively. The result for the uncoated MRR $(N=$ 0 ) is also shown for comparison.

is critical for optimizing the FWM performance. Note that in our calculation we neglect the influence of power-dependent loss on the round-trip field transmission factor $A$, since accounting for it would only lead to a maximum difference in $\Gamma<0.7 \%$.

For a fixed input power $P_{C W}$, varying the GO film parameters such as layer number $N$ and coating length $L_{c}$ changes the intracavity loss and hence intracavity power $P_{\text {intra }}$. Therefore, the power dependent propagation loss and nonlinear parameters of the hybrid MRRs are also affected by $N$ and $L_{c}$. Fig. 4(a) shows $P_{\text {intra }}$ versus $L_{c}$ for the hybrid MRRs with films including (i) $1-5$ and (ii) $10-50$ layers of GO. The other parameters are kept constant: $t=0.912$ and $P_{C W}=$ $25 \mathrm{dBm}$ - taken from our previous experiments [18]. To clearly show the difference, we choose different ranges for $L_{c}$ in Figs. 4(a-i) and (a-ii) - with a smaller range for thicker films $(N \geq 10)$. As can be seen, $P_{\text {intra }}$ decreases with $L_{c}$ and $N$, resulting from an increased intracavity loss in the hybrid MRRs. Figs. 4(b) and (c) show the corresponding propagation
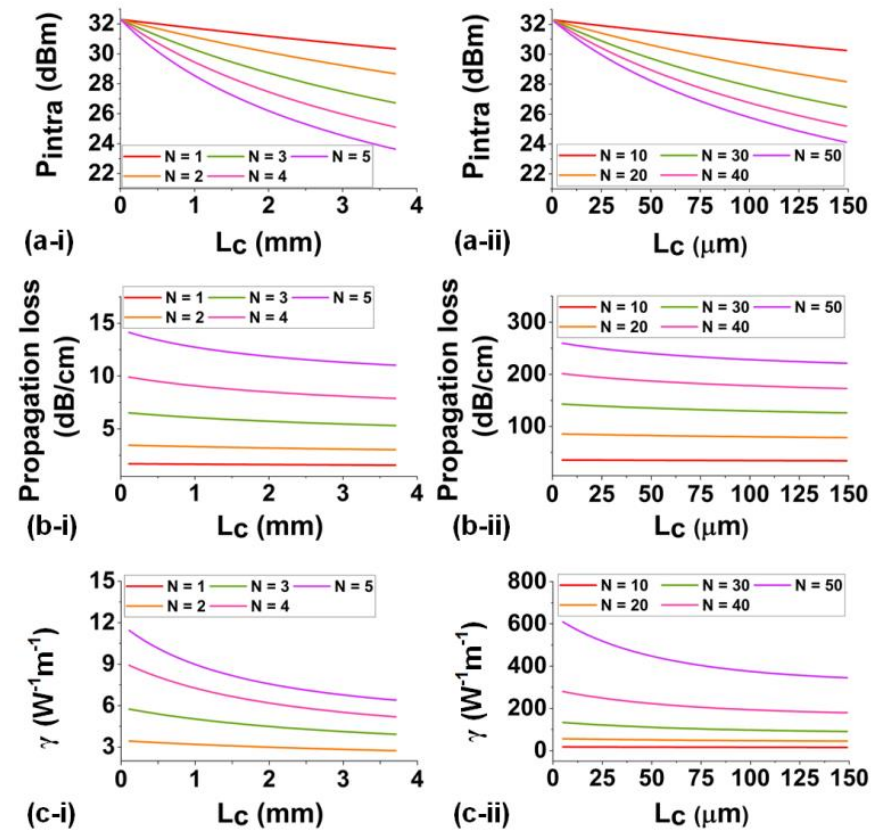

Fig. 4. (a) Intracavity power $P_{\text {intra }}$, (b) propagation loss, and (c) nonlinear parameters $\gamma$ versus coating length $L_{c}$ for the hybrid MRRs with films including (i) $N=1-5$ and (ii) $N=10-50$ GO layers, respectively. In (a) (c), $t=0.912, R=592 \mu \mathrm{m}$, and $P_{C W}=25 \mathrm{dBm}$.

loss and nonlinear parameters $\gamma$ versus $L_{c}$, respectively. Both the propagation loss and nonlinear parameters $\gamma$ decrease with $L_{c}$, showing a trend similar to that of $P_{\text {intra }}$ in Fig. 4(a) and reflecting that the power dependent propagation loss and nonlinear parameters of the hybrid MRRs is strongly dependent on $P_{\text {intra }}$.

\section{OPTIMIZING FWM PERFORMANCE}

In this section, we investigate the influence of the GO film parameters $\left(N, L_{c}\right)$ and MRR coupling strength $(t)$ on the FWM performance of the GO-coated MRRs, taking into account the power-dependent propagation loss and nonlinear parameter discussed in Section III.

The FWM CE of the GO-coated MRRs $\left(C E_{M R R}\right)$ is calculated by $[23,43]$

$$
C E_{M R R}=\frac{P_{\text {idler, out }}}{P_{\text {signal, in }}}=C E_{W G} \cdot F E_{p}^{4} \cdot F E_{s}^{2} \cdot F E_{i}^{2}
$$

TABLE II

PROCESS FLOW TO CALCULATE FWM CE IN GO-COATED MRRS

\begin{tabular}{|c|c|c|c|}
\hline Pre-step ${ }^{\text {a }}$ & Aim & Method \& Theory & Used parameters \\
\hline 0 & Fit $\gamma\left(P_{\text {intra }}\right), \alpha_{c}\left(P_{\text {intra }}\right)$ based on the experimental results in Ref. [18] & Matlab & Measured $\gamma$ and $\alpha_{c}$ in Ref. [18] \\
\hline Step & Aim & Method \& Theory & Used parameters \\
\hline 1 & Calculate round-trip transmission $A\left(N, L_{c}, \alpha_{c}\right)$ & Eq. (2) & $N, L_{c}, \alpha_{c}$ \\
\hline 2 & Calculate build-up factor $\Gamma(A, t)$ & Eq. (1) & $t$ and results in Step 1 \\
\hline 3 & Calculate intracavity power $P_{\text {intra }}\left(\Gamma, P_{p}, P_{s}\right)$ & Eq. (1) & $P_{p}, P_{s}$, and results in Step 2 \\
\hline 4 & Calculate $\gamma\left(P_{\text {intra }}\right)$ and $\alpha_{c}\left(P_{\text {intra }}\right)$ & Matlab & Results in Pre-Step and Step 3 \\
\hline 5 & Calculate CE of GO-coated MRRs b & $\begin{array}{c}\text { Matlab } \\
\text { Eqs. }(3)-(5)\end{array}$ & $t, \kappa$, and results in Step 1,4 \\
\hline
\end{tabular}

a The pre-step is done in Fig. 3 of Section III.

b.To optimize FWM CE, Step $1-5$ were repeated to calculate the CEs for the hybrid MRRs with various $N, L c$, and $t$. 

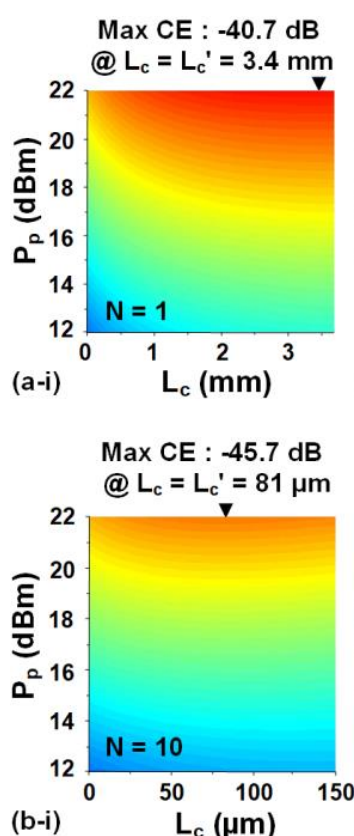
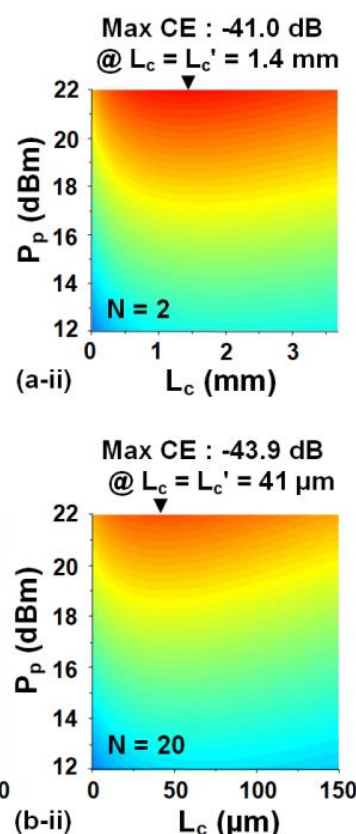
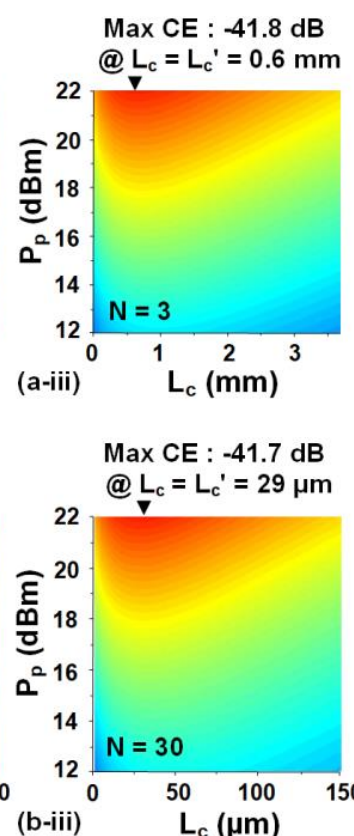
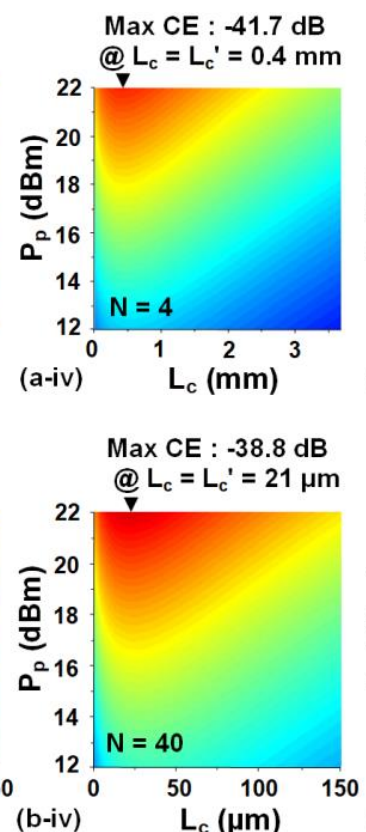
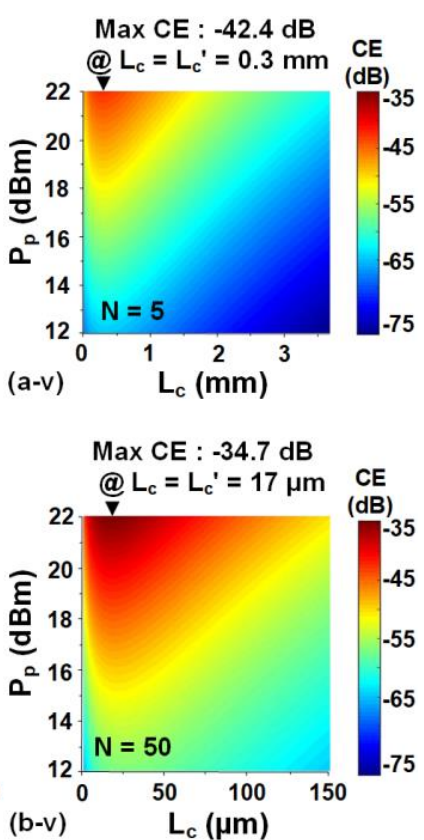

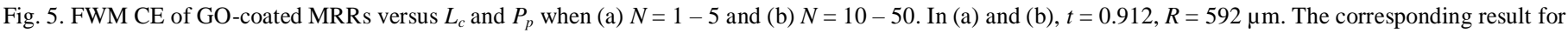
the uncoated MRR (when $L_{c}=0 \mathrm{~mm}$ ) is also shown.

where $P_{\text {idler, out }}$ and $P_{\text {signal, in }}$ are the output power of the idler and input power of the signal, respectively. $C E_{W G}$ is the $\mathrm{CE}$ of an equivalent waveguide with the same length as the circumference of the MRR. The calculation of $C E_{W G}$ is based on the theory in Refs. [13, 17]. For the MRRs with patterned GO films, $C E_{W G}$ is calculated by dividing the equivalent waveguides into coated and uncoated segments that have different propagation loss and nonlinear parameters. $F E_{p, s, i}$ in Eq. (3) are resonant field enhancement factors for the pump, signal, and idler, respectively, which can be expressed as [18, 22, 44]:

$$
F E_{p, s, i}=\kappa \cdot t /\left[1-t^{2} \cdot A \cdot \exp \left(j \cdot \phi_{p, s, i}\right)\right]
$$

where $t$ and $\kappa$ are the field transmission and coupling coefficients defined in Table I, respectively. $\phi_{p, s, i}$ are the round-trip phase shift of the pump, signal, and idler, respectively, which can be given by:

$$
\phi_{p, s, i}=k_{p u, s u, i u} L_{u}+k_{p c, s c, i c} L_{c}
$$

$k_{p c, s c, i c}$ and $k_{p u, s u, i u}$ are the wavenumbers of the pump, signal, and idler for the GO coated and uncoated segments, respectively. Table II summarizes the process flow to calculate the FWM CE of the hybrid MRRs. On the basis of the fit propagation loss and nonlinear parameter in Fig. 3, five steps were repeated to calculate the $\mathrm{CE}$ of hybrid MRRs with different GO film parameters $\left(N, L_{c}\right)$ and coupling strength $(t)$.

We first analyze the FWM CE of the hybrid MRRs with a fixed coupling strength $(t)$ but different GO film parameters $\left(N, L_{c}\right)$. The calculated FWM CE versus coating length $L_{c}$ and input pump power $P_{p}$ is shown in Fig. 5, with (a-i) - (a-v) and (b-i) - (b-v) showing the results for $N=1-5$ and $N=10-50$, respectively. Similar to Fig. 4, a smaller range of $L_{c}$ is chosen for thicker films $(N \geq 10)$ to clearly show the difference. To simplify the discussion, we used the same power for the pump and signal in our calculation, therefore $12-22 \mathrm{dBm}$ of $P_{p}$ in

TABLE III

\begin{tabular}{|c|c|c|c|c|c|c|c|c|}
\hline \multirow{2}{*}{$N$} & \multicolumn{4}{|c|}{ Experimental results a } & \multicolumn{4}{|c|}{ Optimized $L_{c}{ }^{\prime}$ for fixed $t=0.912$} \\
\hline & $L_{c}(\mathrm{~mm})$ & $t$ & $\operatorname{Max} \mathrm{CE}(\mathrm{dB})$ & Max CE enhancement (dB) & $L_{c}{ }^{\prime}(\mathrm{mm})$ & $t$ & $\operatorname{Max} C E(d B)$ & Max CE enhancement (dB) \\
\hline 0 & 0 & 0.912 & -48.4 & 0 & 0 & 0.912 & -48.4 & 0 \\
\hline 1 & \multirow{5}{*}{3.67} & \multirow{5}{*}{0.912} & $\begin{array}{l}-40.8 \\
\end{array}$ & 7.6 & 3.4 & \multirow{5}{*}{0.912} & $\begin{array}{l}-40.7 \\
\end{array}$ & 7.7 \\
\hline 2 & & & -43.1 & 5.3 & 1.4 & & -41.0 & 7.4 \\
\hline 3 & & & -49.1 & -0.7 & 0.6 & & -41.8 & 6.6 \\
\hline 4 & & & -54.9 & -6.5 & 0.4 & & -41.7 & 6.7 \\
\hline 5 & & & -60.9 & -12.5 & 0.3 & & -42.4 & 6.0 \\
\hline 10 & \multirow{5}{*}{0.05} & \multirow{5}{*}{0.912} & $\begin{array}{l}-45.9 \\
\end{array}$ & 2.5 & 0.081 & \multirow{5}{*}{0.912} & $\begin{array}{l}-45.7 \\
\end{array}$ & 2.7 \\
\hline 20 & & & -43.8 & 4.6 & 0.041 & & -43.9 & 4.5 \\
\hline 30 & & & -42.5 & 5.9 & 0.029 & & -41.7 & 6.7 \\
\hline 40 & & & -40.7 & 7.7 & 0.021 & & -38.8 & 9.6 \\
\hline 50 & & & -38.1 & 10.3 & 0.017 & & -34.7 & 13.7 \\
\hline
\end{tabular}

COMPARISON OF HYBRID MRRS WITH OPTMIZED GO COATING LENGTHS AND THOSE IN PREVIOUS EXPERIMENT

${ }^{\text {a }}$ The experimental results are based on the measured values at $P_{p}=P_{s}=22 \mathrm{dBm}$ in Ref. [18]. 

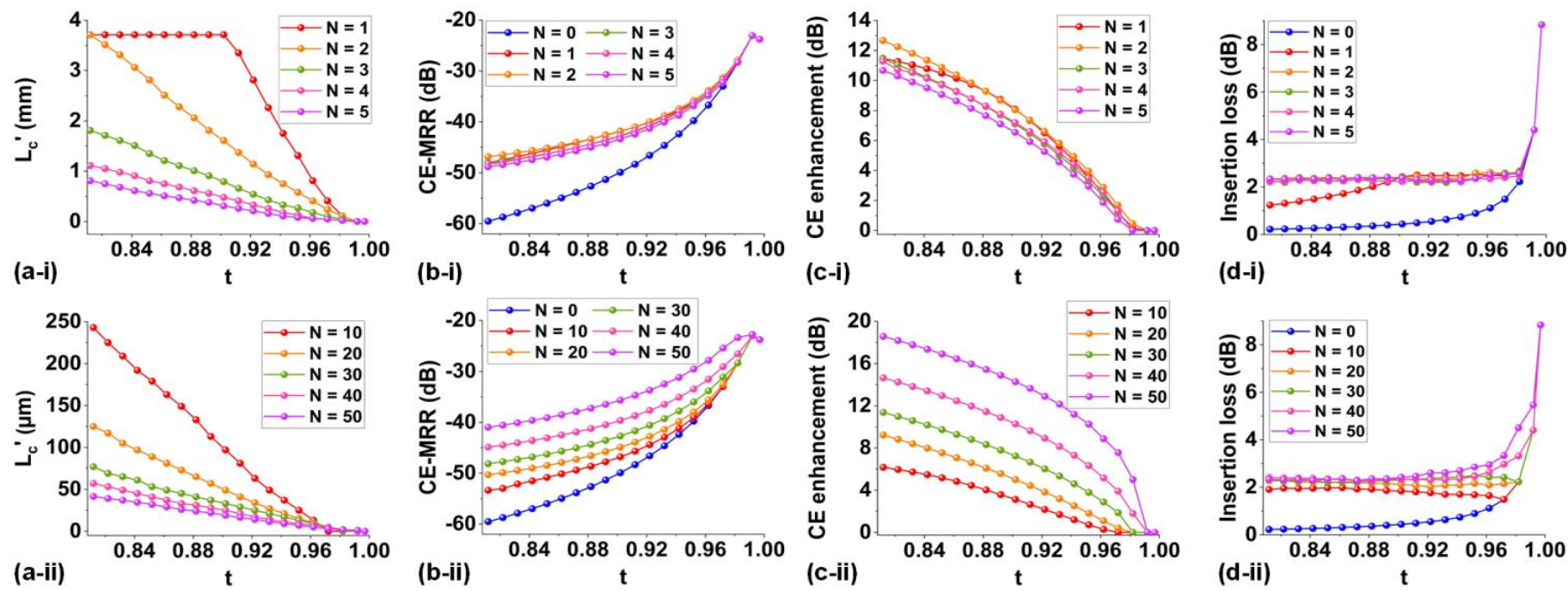

Fig. 6. (a) Optimized coating length $L_{c}^{\prime}$ versus $t$. (b) CE of hybrid MRRs with the optimized coating length $L_{c}^{\prime}$ in (a). (c) CE enhancement of the hybrid MRRs extracted from (b). (d) Insertion loss of the hybrid MRRs with the optimized coating lengths $L_{c}^{\prime}$ in (a). In (a) - (d), $R=592 \mu \mathrm{m}, P_{p}=P_{s}=22 \mathrm{dBm}$, (i) and (ii) show the results for $N=1-5$ and $N=10-50$, respectively. In (b) and (d), the results for the uncoated MRRs $(N=0)$ are also shown for comparison.

Fig. 5 corresponds to $15-25 \mathrm{dBm}$ of $P_{c w}$ in Fig. 3. In our calculation, we used constant $t=0.912$ and $R=592 \mu \mathrm{m}$. The corresponding result for the uncoated MRR (when $L_{\mathrm{c}}=0 \mathrm{~mm}$ ) is also shown for comparison, which achieves the maximum CE of $-48.4 \mathrm{~dB}$ at $P_{p}=P_{s}=22 \mathrm{dBm}$.

In Fig. 5, the $\mathrm{CE}$ of the hybrid MRRs first increases with GO film length $L_{c}$ and then decreases, achieving maximum values at intermediate film lengths. The optimized film length $L_{c}{ }^{\prime}$ that corresponds to the maximum CE decreases with $N$. This reflects the fact that the Kerr nonlinearity enhancement dominates for the devices with relatively small $L_{c}$ and $N$, and the influence of loss increase becomes more significant as $L_{c}$ and $N$ increase.

In Table III, we compare the calculated CE of the hybrid MRRs with optimized GO film lengths and the measured CE in our previous experiment where we fabricated devices with fixed film coating lengths of $\sim 3.67 \mathrm{~mm}$ (i.e., the circumference of the MRR) for $N=1-5$ and $50 \mu \mathrm{m}$ for $N=$ $10-50$ [18]. For the devices with optimized GO film lengths, there is an improvement in the $\mathrm{CE}$ for all the considered GO layer numbers. Particularly, a maximum $\mathrm{CE}$ of $-34.7 \mathrm{~dB}$ is achieved for $N=50$ and $L_{c}=17 \mu \mathrm{m}$, which corresponds to a $\mathrm{CE}$ enhancement of $13.7 \mathrm{~dB}$ compared to the uncoated MRR and $3.4 \mathrm{~dB}$ further improvement relative to previous experimental result.

In addition to GO film parameters $\left(N, L_{c}\right)$, the MRR coupling strength $(t)$ also significantly affects the FWM performance of the hybrid MRRs. Based on the process flow in Table II, we further calculate the FWM CE of hybrid MRRs with different coupling strength $(t)$. In our calculations, we chose 20 different values of $t$ ranging from 0.812 to 0.997 . For each of them, the calculation processes for Fig. 5 with a fixed $t$ were repeated to obtain the optimized film length $L_{c}{ }^{\prime}$ and the corresponding maximum $\mathrm{CE}$ for different numbers of $\mathrm{GO}$ layers $N$.

Fig. 6(a) shows the calculated $L_{c}{ }^{\prime}$ versus $t$, (i) for $N=1-5$ and (ii) for $N=10-50$. The other device parameters are kept the same, i.e., $R=592 \mu \mathrm{m}$ and $P_{p}=P_{s}=22 \mathrm{dBm}$. As can be seen, $L_{\mathrm{c}}$ ' decreases with $t$. This reflects that the positive impact of the GO films in improving the FWM CE decreases with $t$. Fig. 6(b) shows the maximum $\mathrm{CE}$ of the hybrid MRRs corresponding to the calculated $L_{\mathrm{c}}$ ' in Fig. 6(a). The results for the uncoated MRRs $(N=0)$ are also shown for comparison. The CE enhancement compared to the uncoated MRR is further extracted from Fig. 6(b) and plot in Fig. 6(c). A maximum $\mathrm{CE}$ enhancement of $18.6 \mathrm{~dB}$ is achieved at $t=0.812$, $L_{c}=42 \mu \mathrm{m}$, and $N=50$, which is $4.9 \mathrm{~dB}$ higher than the maximum CE enhancement when $t=0.912$. This reflects the fact that reducing $t$ further yields a better CE enhancement. The difference in CE between the hybrid and uncoated MRRs becomes smaller as $t$ increases, which is consistent with the trend for $L_{\mathrm{c}}{ }^{\prime}$ in Fig. 6(a). When $t$ is close to 1, the CE enhancement approaches zero, indicating that incorporating GO films would not bring any benefits in improving the FWM performance in this case. In Fig. 6(d), we plot the insertion loss (at the drop port) of the hybrid MRRs with optimized film lengths $L_{c}^{\prime}$ in Fig. 6(a). It can be seen that the insertion loss increases with $t$ and becomes $>8 \mathrm{~dB}$ when $t$ is close to 1 , which is mainly induced by the four-port MRRs with two identical directional couplers. This indicates that despite the MRR with a weak coupling strength (i.e., high $t$ ) has a high $\mathrm{CE}$, it suffers from a high insertion loss that limits their use in practical applications.

\section{DISCUSSION}

In this section, we discuss the influence of photo-thermal changes in the GO films as well as the effect of varying some of the other MRR paramteres such as ring radius and waveguide dispersion on the FWM performance. This, together with the analysis in Section IV, provides a systematic approach for designing GO-coated MRRs in order to optmize the FWM performance.

As discussed in Section III, photo-thermal changes in the GO films lead to power-dependent propagation loss and 

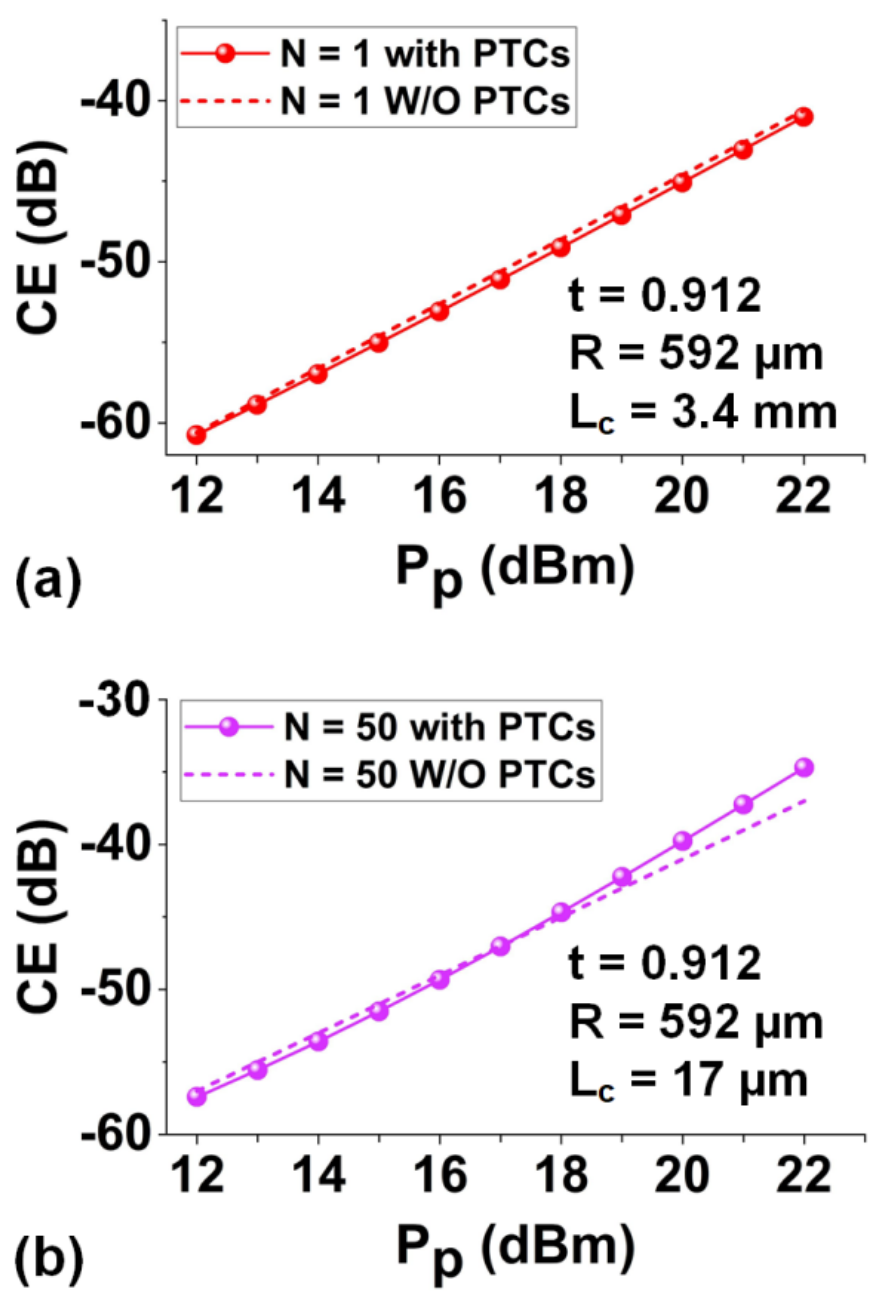

Fig. 7. CE comparison of GO-coated MRRs with and without (W/O) considering photo-thermal changes (PTCs) in GO films when (a) $N=1, L_{c}=$ $3.4 \mathrm{~mm}$ and (b) $N=50, L_{c}=17 \mu \mathrm{m}$. In (a) and (b), $t=0.912$ and $R=592 \mu \mathrm{m}$.

nonlinear parameter $\gamma$ for the hybrid MRRs. Both of these parameters affect the FWM CE and there is a trade-off between them. In Fig. 7, we compare the FWM CE of the hybrid MRRs with and without considering any photo-thermal changes. For the hybrid MRRs without any photo-thermal changes, we used a constant propagation loss and nonlinear parameter, equivalent to their values at low powers. In Figs. 7(a) and (b), we show the results for the hybrid MRRs with 1 and 50 layers of GO. For each of them, optimized film lengths were chosen. The other device parameters are kept the same, i.e., $t=0.912$ and $R=592 \mu \mathrm{m}$. In Fig. 7(a), after including photo-thermal changes, the $\mathrm{CE}$ decreases, with a more notable difference occurring at higher powers. This reflects the fact that the influence of an increase in loss is more significant than the increase of $\gamma$ for the device with a thin GO film. In Fig. 7(b), the CE obtained when including photo-thermal effects is lower at low pump powers, while as the pump power increases, it gradually overtakes the CE obtained without including photo-thermal effects. This reflects a more complex influence of the photo-thermal changes on the FWM performance for the hybrid MRRs with thick GO films, which can be attributed to an increase of defects and imperfect contact as well as more obvious thermal dissipation issue in the thick GO films.

Due to the resonant enhancement effect in the MRRs, the FWM CE can be significantly improved in GO-coated MRRs as compared with GO-coated waveguides. In Fig. 8, we compare the FWM CE of GO-coated MRRs and comparable GO-coated waveguides, (i) for the devices with 1 layer of GO and (ii) for the devices with 50 layers of GO. Similar to the case of Fig. 7, optimized film lengths were chosen for the hybrid MRRs and the other device parameters are kept the same as those in Fig. 7. The hybrid waveguides have the same length as the circumference of the MRRs, and both the MRR and the waveguides have the same GO film length. For the hybrid waveguides, we neglect the slight variation induced by photo-thermal changes in the GO films. As can be seen, the CEs of the hybrid MRRs are much higher than those of the hybrid waveguides for both $N=1$ and $N=50$, clearly reflecting the huge $\mathrm{CE}$ improvement enabled by the resonant structure.

For practical device fabrication, hybrid MRRs with uniformly coated GO films are easier to be fabricated since they do not need lithography or lift-off processes for film patterning. In Fig. 9, we further investigate the FWM performance of these hybrid MRRs. Fig. 9(a-i) shows the
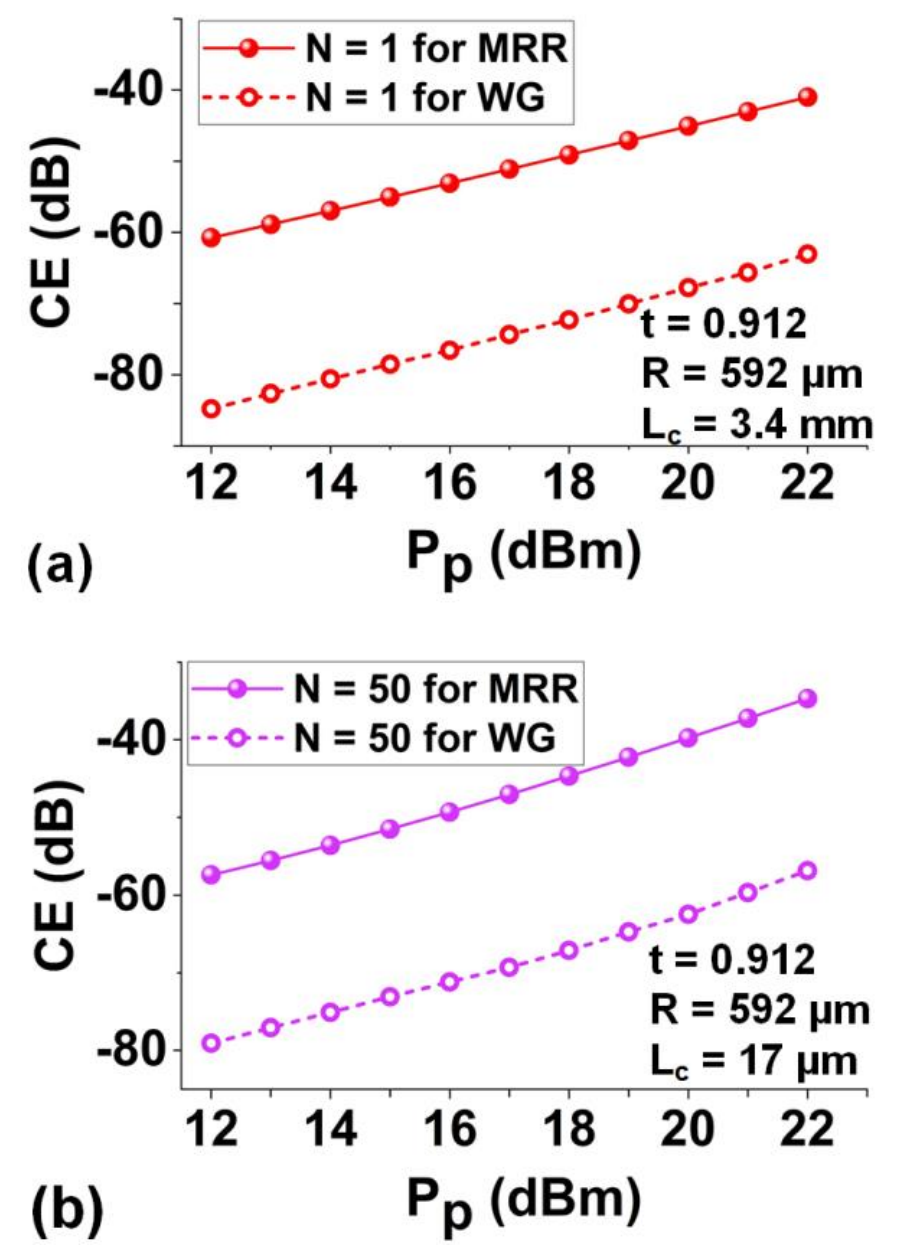

Fig. 8. CE comparison of GO-coated MRRs and comparable GO-coated waveguides when (a) $N=1, L_{c}=3.4 \mathrm{~mm}$ and (b) $N=50, L_{c}=17 \mu \mathrm{m}$. In (a) and (b), $t=0.912$ and $R=592 \mu \mathrm{m}$. WG: waveguide. 

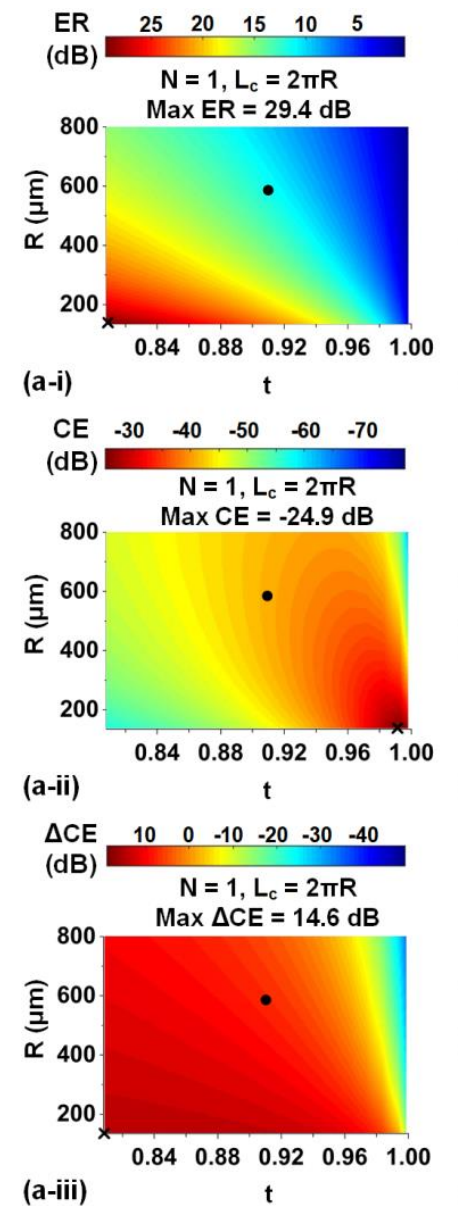

Fig. 9. Performance comparison of uniformly GO-coated MRRs when (a) $N$ $=1$ and (b) $N=2$. In (a) - (b), $P_{p}=P_{s}=22 \mathrm{dBm}$, (i) - (iii) show the ER, CE, and CE enhancement versus $R$ and $t$, respectively. The black circles mark the experimental results in Ref. [18] and the black crosses mark the maximum values in each figure. $\triangle \mathrm{CE}$ : $\mathrm{CE}$ enhancement compared to uncoated MRRs with the same $t$ and $R$.

MRR's ER versus its radius $R$ and coupling strength $t$ when $N$ $=1$ and $P_{p}=P_{s}=22 \mathrm{dBm}$. The ER decreases with both $R$ and $t$ - the former results from the increase of the intracavity loss with $R$, while the latter is consistent with the trend in Fig. 2(b). Fig. 9(a-ii) shows the CE versus $R$ and $t$. The CE enhancement relative to the uncoated MRR is further extracted from Fig. 9(a-ii) and shown in Fig. 9(a-iii). In our calculation, we neglect the slight difference in the MRR coupling strength $t$ between the GO coated and uncoated MRRs, since including it would result in a difference of only $<0.3 \%$. In Fig. 9(a-ii), the $\mathrm{CE}(-40.8 \mathrm{~dB})$ at $R=592 \mu \mathrm{m}$ and $t=0.912$ is marked, which corresponds to a $\mathrm{CE}$ enhancement of $7.6 \mathrm{~dB}$ in Fig. 9(a-iii), showing good agreement with the experimental result in Ref. [18]. The maximum CE $(-24.9 \mathrm{~dB})$ at $R=135 \mu \mathrm{m}$ and $t=$ 0.992 is also marked, which is $15.9 \mathrm{~dB}$ higher than the $\mathrm{CE}$ at $R$ $=592 \mu \mathrm{m}$ and $t=0.912$ and corresponds to a CE enhancement of $-1.8 \mathrm{~dB}$. In Fig. 9(a-iii), a maximum CE enhancement of $14.6 \mathrm{~dB}$ is achieved at $R=135 \mu \mathrm{m}$ and $t=0.812$, which is different to the point corresponding to the maximum CE. This reflects the trade-off between achieving the maximum $\mathrm{CE}$ versus the maximum relative $\mathrm{CE}$ enhancement for the device design, which is consistent with the results in Figs. 6(b) and
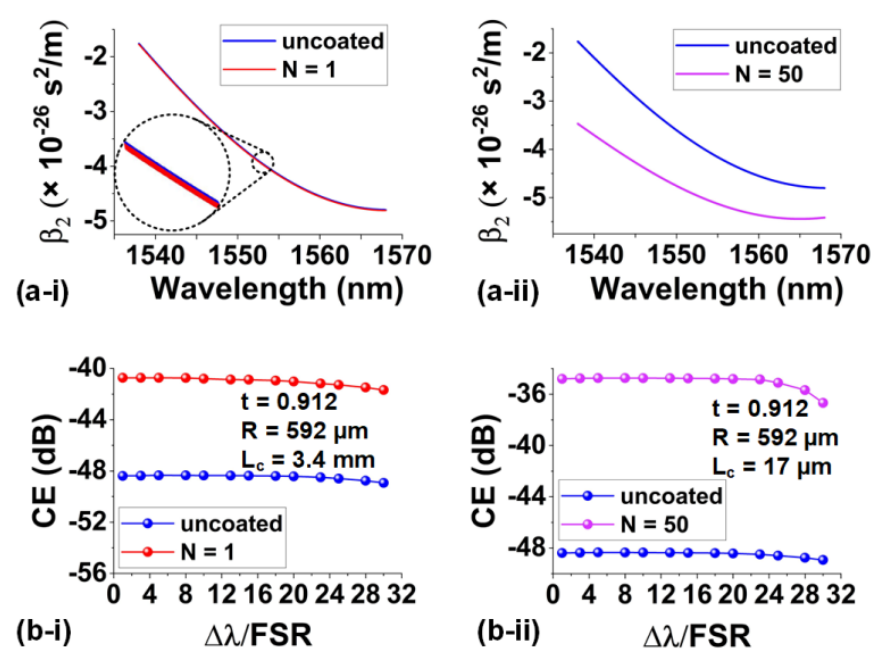

Fig. 10. (a) Group-velocity dispersion $\beta_{2}$ for hybrid MRRs with (i) $N=1$ and (ii) $N=50$ layers of GO. (b) CE versus $\Delta \lambda /$ FSR for hybrid MRRs when (i) $N$ $=1, L_{c}=3.4 \mathrm{~mm}$ and (ii) $N=1, L_{c}=17 \mu \mathrm{m}$. In (a) and (b), $t=0.912, R=$ $592 \mu \mathrm{m}$, and $P_{p}=P_{s}=22 \mathrm{dBm}$. The corresponding results for the uncoated MRR are also shown for comparison.

(c). Fig. 9(b) shows the corresponding results for $N=2$. The maximum $\mathrm{CE}$ enhancement is improved further by $\sim 4.3 \mathrm{~dB}$ as compared with that for $N=1$, while both the maximum ER and $\mathrm{CE}$ decrease due to the increase in loss with film thickness. This, on one hand, indicates that a high $\mathrm{CE}$ enhancement can be achieved for the hybrid MRRs with small radii even without the use of film patterning, while on the other hand, it reflects the fact that the CE significantly decreases with GO film thickness for the uniformly coated MRRs.

Finally, we investigate the influence of waveguide dispersion on the FWM performance of hybrid MRRs. Fig. 10 (a) shows the group-velocity dispersion $\beta_{2}$ for the hybrid MRRs with (i) $N=1$ and (ii) $N=50$ layers of GO, together with the $\beta_{2}$ of the uncoated MRR. The material dispersion of GO and doped silica was taken from Refs. [12, 27]. The $\beta_{2}$ of the hybrid MRRs is slightly lower as compared with the uncoated MRR, with the difference becoming more significant for the device with thicker films. The reduced $\beta_{2}$ induced by the GO films yields an enhanced anomalous dispersion and consequently better phase matching for FWM [45]. Fig. 10(b) shows the CE versus $\Delta \lambda$ (defined as wavelength spacing between pump and signal) for the hybrid MRRs, (i) for $N=1$, $L_{c}=3.4 \mathrm{~mm}$ and (ii) for $N=50, L_{c}=17 \mu \mathrm{m}$. The corresponding result for the uncoated MRR is also shown for comparison. The other parameters are kept the same as $t=$ 0.912 and $R=592 \mu \mathrm{m}$. The CE slightly decreases with $\Delta \lambda$, with a difference $<2 \mathrm{~dB}$ for $\Delta \lambda / \mathrm{FSR}=30$ when $N=50, L_{c}=$ $17 \mu \mathrm{m}$. This reflects the fact that both the doped silica and the GO film have a low material dispersion, which allows highly effective phase matching for broadband FWM.

These results could ultimately have significant implications for realizing microcomb devices with lower threshold powers for many applications including microwave photonics [46-78], microcombs generally [79-81] and other nonlinear 
applications [82-84]. MRRs are also very useful in terms of dispersion management and compensation devices [85-89].

\section{CONCLUSION}

In summary, the FWM performance of MRRs integrated with 2D layered GO films is theoretically studied and optimized based on material and device parameters from previous experiments. A detailed analysis for the influence of GO film parameters and MRR coupling strength on the FWM $\mathrm{CE}$ of the GO-coated MRRs is performed. By redesigning the device parameters to properly balance the trade-off between the Kerr nonlinearity and loss, up to $\sim 18.6 \mathrm{~dB}$ enhancement in the FWM CE is achieved, corresponding to $\sim 8.3 \mathrm{~dB}$ further improvement over what was achieved experimentally. The influence of photo-thermal changes in the GO films as well as the variation of some other MRR parameters such as ring radius and waveguide dispersion is also investigated. These results confirm the effectiveness of introducing GO films to improve the MRR's FWM performance and serve as a roadmap for optimizing the FWM performance of GO-coated MRRs.

\section{Competing interests: The authors declare no competing interests.}

\section{REFERENCES}

1. K. Loh et al., "Graphene oxide as a chemically tunable platform for optical applications," Nat. Chem., vol. 2, no. 12, pp. 1015-1024, Dec. 2010.

2. J. Wu, L. Jia, Y. Zhang, Y. Qu, B. Jia, and D. Moss, "Graphene oxide for integrated photonics and flat optics," Adv. Mater., vol. 33, no. 3, pp. 200415, Jan. 2021.

3. A. Dideikin and A. Vul, "Graphene oxide and derivatives: the place in graphene family," Front. Phys.," vol. 6, no. 149, pp. 1-29, Dec. 2019.

4. Y. Zhang et al., "Photoreduction of graphene oxides: methods, properties, and applications," Adv. Opt. Mater., vol. 2, no. 1, pp. 10-28, Oct. 2014.

5. X. Zheng et al., "In situ third-order non-linear responses during laser reduction of graphene oxide thin films towards on-chip nonlinear photonic devices," Adv. Mater., vol. 26, no. 17, pp. 2699-2703, Mar. 2014.

6. X. Xu et al., "Observation of third-order nonlinearities in graphene oxide film at telecommunication wavelengths," Sci. Rep., vol. 7, no. 1, pp. 9646, Aug. 2017.

7. J. Ren et al., "Giant third-order nonlinearity from low-loss electrochemical graphene oxide film with a high power stability," App. Phys. Lett., vol. 109, no. 22, pp. 221105, Nov. 2016.

8. I. Antoine et al., "Second harmonic scattering from mass characterized 2D graphene oxide sheets," Chem. Comm., vol. 56, no. 27, pp. 38593862, Feb. 2020.

9. X. Jiang et al., "Graphene oxides as tunable broadband nonlinear optical materials for femtosecond laser pulses," J. Phys. Chem. Lett., vol. 3, no. 6, pp. 785-790, Mar. 2012.

10. B. Eggleton, B. Davies, and K. Richardson, "Chalcogenide photonics," Nat. Photonics, vol. 5, no. 3, pp. 141-148, Feb. 2011.

11. L. Guo et al., "Bandgap tailoring and synchronous microdevices patterning of graphene oxides," J. Phys. Chem. C, vol. 116, no. 5, pp. 3594-3599, Jan. 2012.

12. Y. Yang et al., "Graphene-based multilayered metamaterials with phototunable architecture for on-chip photonic devices," ACS Photonics, vol.6, no. 4, pp. 1033-1040, Feb. 2019.

13. Y. Yang et al., "Invited article: enhanced four-wave mixing in waveguides integrated with graphene oxide," APL Photonics, vol. 3, no. 12, pp. 120803, Oct. 2018.

14. Y. Zhu et al., "Graphene and graphene oxide: synthesis, properties, and applications," Adv. Mater., vol. 22, no. 35, pp. 3906-3924, Oct. 2010.
15. Y. Zhang et al., "Optimizing the Kerr nonlinear optical performance of silicon waveguides integrated with 2D graphene oxide films," $J$. Lightwave Technol., doi: 10.1109/JLT.2021.3069733.

16. Y. Qu et al., "Analysis of four-wave mixing in silicon nitride waveguides integrated with 2D layered graphene oxide films," J. Lightwave Technol., vol. 39, no. 9, pp. 2902-2910, May. 2021.

17. Y. Qu et al., "Enhanced four-wave mixing in silicon nitride waveguides integrated with 2D layered graphene oxide films," Adv. Opt. Mater., vol. 8, no. 20, pp. 2001048 , Oct. 2020.

18. J. Wu et al., " $2 \mathrm{D}$ layered graphene oxide films integrated with micro-ring resonators for enhanced nonlinear optics," Small, vol. 16, no. 16, pp. 1906563, Mar. 2020.

19. Y. Zhang et al., "Enhanced Kerr nonlinearity and nonlinear figure of merit in silicon nanowires integrated with 2D graphene oxide films," ACS Appl. Mater. Interfaces, vol. 12, no. 29, pp. 33094-33103, Jun. 2020

20. J. Lee et al., "A Q-switched, mode-locked fiber laser using a graphene oxide-based polarization sensitive saturable absorber," Laser Phys. Lett., vol. 10, no. 3, pp. 035103, Jan. 2013.

21. G. Fernandes et al., "Field-controllable second harmonic generation at a graphene oxide heterointerface," Nanotechnol., vol. 29, no. 10, pp. 105201, Jan. 2018.

22. M. Ferrera et al., "Low-power continuous-wave nonlinear optics in doped silica glass integrated waveguide structures," Nat. Photonics, vol. 2, no. 12, pp. 737-740, Nov. 2008.

23. P. Absil et al., "Wavelength conversion in GaAs micro-ring resonators," Opt. Lett., vol. 25, no. 8, pp. 554-556, Apr. 2000.

24. F. Bonaccorso et al., "Graphene photonics and optoelectronics," Nat. Photonics, vol. 4, no. 9, pp. 611-622, Aug. 2010.

25. D. Moss, R. Morandotti, A. Gaeta, and M. Lipson, "New CMOScompatible platforms based on silicon nitride and Hydex for nonlinear optics," Nat. Photonics, vol. 7, no. 8, pp. 597-607, Jul. 2013.

26. L. Razzari et al., "CMOS-compatible integrated optical hyper-parametric oscillator," Nat. Photonics, vol. 4, no. 1, pp. 41-45, Dec. 2010.

27. X. Xu et al., "11 TOPS photonic convolutional accelerator for optical neural networks," Nature, vol. 589, no. 7840, pp. 44-51, Jan. 2021.

28. J. Wu et al., "Graphene oxide waveguide and micro-ring resonator polarizers," Laser Photonics Rev., vol. 13, no. 9, pp. 1900056, Aug. 2019.

29. Q. Feng et al., "Enhanced optical Kerr nonlinearity of graphene/Si hybrid waveguide," Appl. Phys. Lett., vol. 114, no. 7, pp. 071104, Feb. 2019.

30. L. Liu et al., "Enhanced optical Kerr nonlinearity of MoS_2 on silicon waveguides," Photonics Res., vol. 3, no. 5, pp. 206, Oct. 2015.

31. T. Gu et al., "Regenerative oscillation and four-wave mixing in graphene optoelectronics," Nat. Photonics, vol. 6, no. 8, pp. 554-559, Aug. 2012.

32. H. Lin et al., "Chalcogenide glass-on-graphene photonics," Nat. Photonics, vol. 11, no. 12, pp. 798-805, Dec. 2017.

33. W. Chong et al., "Photo-induced reduction of graphene oxide coating on optical waveguide and consequent optical intermodulation," Sci. Rep., vol. 6, no. 1, pp. 23813, Apr. 2016.

34. J. Wang et al., "Optical absorption in graphene-on-silicon nitride microring resonators," IEEE Photon. Technol. Lett., vol. 27, no. 16, pp. 1765-1767, Aug. 2015.

35. C. Vendromin and M. Dignam, "Optimization of a lossy microring resonator system for the generation of quadrature-squeezed states," Phys. Rev. A, vol. 102, no. 2, pp. 023705, Aug. 2020.

36. H. Arianfard et al., "Three waveguide coupled sagnac loop reflectors for advanced spectral engineering," J. Lightwave Technol., doi: 10.1109/JLT.2021.3066256.

37. J. Wu et al., "Nested configuration of silicon microring resonator with multiple coupling regimes," IEEE Photon. Technol. Lett., vol. 25, no. 6, pp. 580-583, Mar. 2013.

38. J. Wu, T. Moein, X. Xu, and D. J. Moss, "Advanced photonic filters based on cascaded Sagnac loop reflector resonators in silicon-oninsulator nanowires," APL Photonics, vol. 3, 046102 (2018). DOI:/10.1063/1.5025833Apr. 2018.

39. J. Wu, T. Moein, X. Xu, G. H. Ren, A. Mitchell, and D. J. Moss, "Microring resonator quality factor enhancement via an integrated Fabry-Perot cavity," APL Photonics, vol. 2, 056103 (2017).

.40. H. Arianfard, J. Wu, S. Juodkazis, and D. J. Moss, "Advanced MultiFunctional Integrated Photonic Filters Based on Coupled Sagnac Loop Reflectors", Journal of Lightwave Technology, Vol. 39, No.5, pp.14001408 (2021). DOI: 10.1109/JLT.2020.3037559.

41. David J. Moss, "Optimization of Optical Filters based on Integrated Coupled Sagnac Loop Reflectors", Research Square (2021). DOI: https://doi.org/10.21203/rs.3.rs-478204/v1 
42. H. Arianfard, J. Wu, S. Juodkazis, D. J. Moss, "Spectral Shaping Based on Integrated Coupled Sagnac Loop Reflectors Formed by a Self-Coupled Wire Waveguide", submitted, IEEE Photonics Technology Letters, vol. 33 (2021).

43. M. Ji et al., "Enhanced parametric frequency conversion in a compact silicon-graphene microring resonator," Opt. Exp., vol. 23, no. 14, pp. 18679-18685, Jul. 2015.

44. M. Ferrera et al., "Low power four wave mixing in an integrated microring resonator with $\mathrm{Q}=1.2$ million," Opt. Exp., vol. 17, no. 16, pp. 14098-14103, Aug. 2009.

45. M. Foster et al., "Broad-band optical parametric gain on a silicon photonic chip," Nature, vol. 441, no. 7096, pp. 960-963, Jun. 2006.

46. Mengxi Tan, X. Xu, J. Wu, T. G. Nguyen, S. T. Chu, B. E. Little, R. Morandotti, A. Mitchell, and David J. Moss, "Photonic Radio Frequency Channelizers based on Kerr Optical Micro-combs", Journal of Semiconductors $\underline{\mathbf{4 2}}$ (4), 041302 (2021). (ISSN 1674-4926). DOI: $10.1088 / 1674-4926 / 42 / 4 / 041302$.

47. H.Bao, L.Olivieri, M.Rowley, S.T. Chu, B.E. Little, R.Morandotti, D.J. Moss, J.S.T. Gongora, M.Peccianti and A.Pasquazi, "Laser Cavity Solitons and Turing Patterns in Microresonator Filtered Lasers: properties and perspectives", Paper No. LA203-5, Paper No. 11672-5, SPIE LASE, SPIE Photonics West, San Francisco CA March 6-11 (2021). DOI: $10.1117 / 12.2576645$

48. Mengxi Tan, X. Xu, J. Wu, A. Boes, T. G. Nguyen, S. T. Chu, B. E. Little, R. Morandotti, A. Mitchell, and David J. Moss, "Advanced microwave signal generation and processing with soliton crystal microcombs", or "Photonic convolutional accelerator and neural network in the Tera-OPs regime based on Kerr microcombs", Paper No. 11689-38, PW21O-OE201-67, Integrated Optics: Devices, Materials, and Technologies XXV, SPIE Photonics West, San Francisco CA March 6-11 (2021). DOI: 10.1117/12.2584017

49. Mengxi Tan, Bill Corcoran, Xingyuan Xu, Andrew Boes, Jiayang Wu, Thach Nguyen, Sai T. Chu, Brent E. Little, Roberto Morandotti, Arnan Mitchell, and David J. Moss, "Optical data transmission at 40 Terabits/s with a Kerr soliton crystal microcomb", Paper No.11713-8, PW21OOE803-23, Next-Generation Optical Communication: Components, SubSystems, and Systems X, SPIE Photonics West, San Francisco CA March 6-11 (2021). DOI:10.1117/12.2584014

50. Mengxi Tan, X. Xu, J. Wu, A. Boes, T. G. Nguyen, S. T. Chu, B. E. Little, R. Morandotti, A. Mitchell, and David J. Moss, "RF and microwave photonic, fractional differentiation, integration, and Hilbert transforms based on Kerr micro-combs", Paper No. 11713-16, PW21OOE803-24, Next-Generation Optical Communication: Components, SubSystems, and Systems X, SPIE Photonics West, San Francisco CA March 6-11 (2021). DOI:10.1117/12.2584018

51. Mengxi Tan, X. Xu, J. Wu, A. Boes, T. G. Nguyen, S. T. Chu, B. E. Little, R. Morandotti, A. Mitchell, and David J. Moss, "Broadband photonic RF channelizer with 90 channels based on a soliton crystal microcomb", or "Photonic microwave and RF channelizers based on Kerr micro-combs", Paper No. 11685-22, PW21O-OE106-49, Terahertz, RF, Millimeter, and Submillimeter-Wave Technology and Applications XIV, SPIE Photonics West, San Francisco CA March 6-11 (2021). DOI: $10.1117 / 12.2584015$

52. X. Xu, M. Tan, J. Wu, S. T. Chu, B. E. Little, R. Morandotti, A. Mitchell, B. Corcoran, D. Hicks, and D. J. Moss, "Photonic perceptron based on a Kerr microcomb for scalable high speed optical neural networks", IEEE Topical Meeting on Microwave Photonics (MPW), pp. 220-224,.Matsue, Japan, November 24-26, 2020. Electronic ISBN:978-4-88552-331-1. 10.23919/MWP48676.2020.9314409

53. Mengxi Tan, Bill Corcoran, Xingyuan Xu, Andrew Boes, Jiayang Wu, Thach Nguyen, S.T. Chu, B. E. Little, Roberto Morandotti, Arnan Mitchell, and David J. Moss, "Ultra-high bandwidth optical data transmission with a microcomb", IEEE Topical Meeting on Microwave Photonics (MPW), pp. 78-82.Virtual Conf., Matsue, Japan, November 24-26, 2020. Electronic ISBN:978-4-88552-331-1. DOI: 10.23919/MWP48676.2020.9314476

54. M. Tan, X. Xu, J. Wu, R. Morandotti, A. Mitchell, and D. J. Moss, "RF and microwave high bandwidth signal processing based on Kerr Microcombs", Advances in Physics X, VOL. 6, NO. 1, 1838946 (2020). DOI: $10.1080 / 23746149.2020 .1838946$.

55. Mengxi Tan, Xingyuan Xu, Jiayang Wu, Thach G. Nguyen, Sai T. Chu, Brent E. Little, Roberto Morandotti, Arnan Mitchell, and David J. Moss, "Photonic Radio Frequency Channelizers based on Kerr Micro-combs and Integrated Micro-ring Resonators", JOSarXiv.202010.0002.
56. Mengxi Tan, Xingyuan Xu, David Moss "Tunable Broadband RF Photonic Fractional Hilbert Transformer Based on a Soliton Crystal Microcomb", Preprints, DOI: 10.20944/preprints202104.0162.v1

57. Mengxi Tan, X. Xu, J. Wu, T. G. Nguyen, S. T. Chu, B. E. Little, R. Morandotti, A. Mitchell, and David J. Moss, "Orthogonally polarized Photonic Radio Frequency single sideband generation with integrated micro-ring resonators", Journal of Semiconductors $\underline{\mathbf{4 2}}$ (4), 041305 (2021). DOI: $10.1088 / 1674-4926 / 42 / 4 / 041305$.

58. T. G. Nguyen et al., "Integrated frequency comb source-based Hilbert transformer for wideband microwave photonic phase analysis," Opt. Express, vol. 23, no. 17, pp. 22087-22097, Aug. 2015.

59. X. Xu et al., "Reconfigurable broadband microwave photonic intensity differentiator based on an integrated optical frequency comb source," APL Photonics, vol. 2, no. 9, 096104, Sep. 2017.

60. X. Xu, M. Tan, J. Wu, R. Morandotti, A. Mitchell, and D. J. Moss, "Microcomb-based photonic RF signal processing", IEEE Photonics Technology Letters, vol. 31 no. 23 1854-1857, 2019.

61. $\mathrm{X}$. $\mathrm{Xu}$, et al., "Advanced RF and microwave functions based on an integrated optical frequency comb source," Opt. Express, vol. 26, no. 3, pp. 2569-2583, Feb. 2018.

62. X. Xu, et al., "Broadband RF channelizer based on an integrated optical frequency Kerr comb source," Journal of Lightwave Technology, vol. 36, no. 19, pp. 4519-4526, 2018.

63. X. Xu, et al., "Continuously tunable orthogonally polarized RF optical single sideband generator based on micro-ring resonators," Journal of Optics, vol. 20, no. 11, 115701. 2018.

64. X. Xu, et al., "Orthogonally polarized RF optical single sideband generation and dual-channel equalization based on an integrated microring resonator," Journal of Lightwave Technology, vol. 36, no. 20, pp. 4808-4818. 2018.

65. X. Xu, et al., "Photonic microwave true time delays for phased array antennas using a $49 \mathrm{GHz}$ FSR integrated optical micro-comb source," Photonics Res, vol. 6, no. 5, pp. B30-B36, 2018.

66. $\mathrm{X}$. Xu, et al., "Advanced adaptive photonic RF filters with 80 taps based on an integrated optical micro-comb source," Journal of Lightwave Technology, vol. 37, no. 4, pp. 1288-1295, 2019.

67. W. Liang, et al., "High spectral purity Kerr frequency comb radio frequency photonic oscillator," Nature Communications, vol. 6 pp. 7957. 2015.

68. J. Liu, et al., "Photonic microwave generation in the X-and $\mathrm{K}$-band using integrated soliton microcombs" Nature Photonics, vol. 14, pp. 1-6, 2020.

69. X. Xu, et al., Broadband microwave frequency conversion based on an integrated optical micro-comb source", Journal of Lightwave Technology, vol. 38 no. 2, pp. 332-338, 2020.

70. M. Tan, et al., "Photonic RF and microwave filters based on $49 \mathrm{GHz}$ and $200 \mathrm{GHz}$ Kerr microcombs", Optics Comm. vol. 465, Article: 125563, Feb. 22. 2020

71. X. Xu, et al., "Broadband photonic RF channelizer with 90 channels based on a soliton crystal microcomb", Journal of Lightwave Technology, Vol. 38, no. 18, pp.5116 - 5121, 2020. doi: 10.1109/JLT.2020.2997699.

72. $\mathrm{X}$. $\mathrm{Xu}$, et al., "Photonic RF and microwave integrator with soliton crystal microcombs", IEEE Transactions on Circuits and Systems II: Express Briefs, Vol.67, Issue: 12, pp. 3582-3586, (2020). DOI:10.1109/TCSII.2020.2995682.

73. $\mathrm{X}$. Xu, et al., "Photonic RF phase-encoded signal generation with a microcomb source", Journal of Lightwave Technology, vol. 38, no. 7, pp. $1722-1727,2020$

74. X. Xu, et al., "High performance RF filters via bandwidth scaling with Kerr micro-combs," APL Photonics, vol. 4, no. 2, pp. 026102. 2019.

75. M. Tan, et al., "Microwave and RF photonic fractional Hilbert transformer based on a $50 \mathrm{GHz}$ Kerr micro-comb", Journal of Lightwave Technology, vol. 37, no. 24, pp. $6097-6104,2019$.

76. M. Tan, et al., "RF and microwave fractional differentiator based on photonics", IEEE Transactions on Circuits and Systems: Express Briefs, Vol. 67, Issue: 11, pp.2767-2771 (2020). DOI:10.1109/TCSII.2020.2965158

77. M. Tan, et al., "Photonic RF arbitrary waveform generator based on a soliton crystal micro-comb source", Journal of Lightwave Technology, vol. 38, (22) Page(s): 6221-6226, Oct 22 (2020). DOI: 10.1109/JLT.2020.3009655.

78. H. Bao, et al., "Laser cavity-soliton microcombs," Nature Photonics, vol. 13, no. 6, pp. 384-389, Jun. 2019. 
79. H. Bao, L. Olivieri, M. Rowley, S. T. Chu, B. E. Little, R. Morandotti, D. J. Moss, J. S. T. Gongora, M. Peccianti and A. Pasquazi, "Turing patterns in a fibre laser with a nested micro-resonator: robust and controllable micro-comb generation", Physical Review Research, vol. 2, pp. 023395, 2020.

80. L. D. Lauro, J. Li, D. J. Moss, R. Morandotti, S. T. Chu, M. Peccianti, and A. Pasquazi, "Parametric control of thermal self-pulsation in microcavities," Opt. Lett. vol. 42, no. 17, pp. 3407-3410, Aug. 2017.

81. H. Bao, et al., "Type-II micro-comb generation in a filter-driven four wave mixing laser," Photonics Research, vol. 6, no. 5, pp. B67-B73, May 2018.

82. T.Monro, D.J.Moss, M. Bazylenko, C. Martijn de Sterke, and L. Poladian, "Observation of self-trapping of light in a self written channel in photosensitive glass", Physical Review Letters, vol. 80, 4072 (1998).

83. M. Rochette, L. Fu, V. Ta'eed, D.J. Moss, B.J. Eggleton, "2R optical regeneration: an all-optical solution for BER improvement", IEEE Journal of Selected Topics in Quantum Electronics, vol. 12, no. 4, 736744 (2006).

84. C Grillet et al.,"Efficient coupling to chalcogenide glass photonic crystal waveguides via silica optical fiber nanowires", Optics Express, vol. 14, no. 3, 1070-1078 (2006).

85. T.Ido et al., IEEE Photonics Technology Letters, Vol. 6, 1207 (1994). DOI: $10.1109 / 68.329640$.

86. D. J. Moss, M. Lamont, S. Mclaughlin, G. Randall, P. Colbourne, S. Kiran and C. A. Hulse,"Tunable dispersion and dispersion slope compensators for $10 \mathrm{~Gb} / \mathrm{s}$ using all-pass multicavity etalons", IEEE Photonics Technology Letters, vol. 15, no. 5, 730-732 (2003). DOI: 10.1109/LPT.2003.809921.

87. L.M. Lunardi, D.Moss, S.Chandrasekhar, L.L.Buhl, A. Hulse, P.Colbourne, G.Randall, S.Mclaughlin, "Tunable dispersion compensators based on multi-cavity all-pass etalons for $40 \mathrm{~Gb} / \mathrm{s}$ systems", Journal of Lightwave Technology, vol. 20, no. 12, 2136 (2002). DOI: 10.1109/JLT.2002.806768.

88. D. J. Moss, et al., "Multichannel tunable dispersion compensation using all-pass multicavity etalons", paper TuT2 Optical Fiber Communications Conference, Anaheim (2002). Post-conference Technical Digest (IEEE Cat. No.02CH37339). Opt Soc. America. Part vol.1, 2002, pp. 132-3. Washington, DC, USA.

89. L.M. Lunardi, D. Moss, S. Chandrasekhar, L.L. Buhl, "An etalon based tunable dispersion compensator (TDC) device for $40 \mathrm{~Gb} / \mathrm{s}$ applications", European Conference on Optical Communications (ECOC), paper 5.4.6, Copenhagen, Sept. (2002). IEEE. Part vol. 2, 2002, pp. 2 vol. 2. Piscataway, NJ, USA. INSPEC Accession Number: 9153476, Print ISBN: 87-90974-63-8. 